\title{
Flavonoid Silibinin Increases Hair-Inductive Property Via Akt and Wnt/ $\beta$-Catenin Signaling Activation in 3-Dimensional-Spheroid Cultured Human Dermal Papilla Cells
}

\author{
Hye In Cheon ${ }^{1}$, Seunghee $\mathrm{Bae}^{2}$, and Kyu Joong $\mathrm{Ahn}^{1 *}$ \\ ${ }^{1}$ Department of Dermatology, Konkuk University School of Medicine, Seoul 05030, Republic of Korea \\ ${ }^{2}$ Research Institute for Molecular-Targeted Drugs, Department of Cosmetics Engineering, Konkuk University, Seoul 05029, Republic of Korea
}

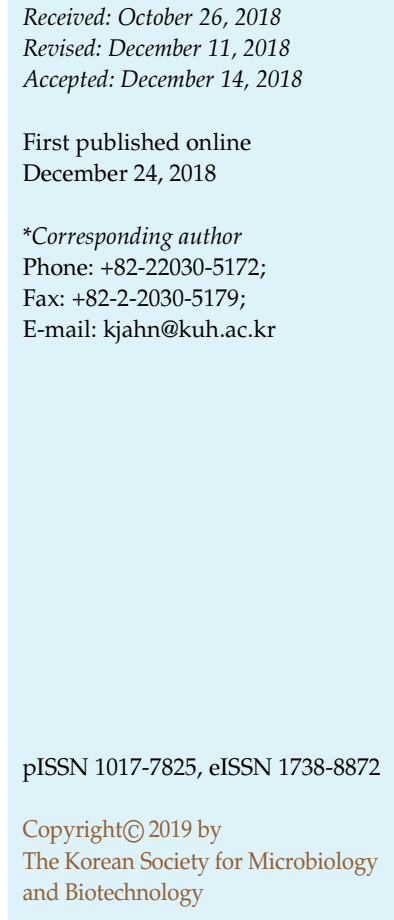

Hair loss, also known as alopecia, is a common dermatological condition of psychosocial significance; development of therapeutic candidates for the treatment of this condition is, hence, important. Silibinin, a secondary metabolite from Silybum marianum, is an effective antioxidant that also prevents various cutaneous problems. In this study, we have investigated the effect of silibinin on hair induction using three-dimensional (3D) cultured, human dermal papilla (DP) spheroids. Silibinin was found to significantly increase viability through AKT serine/threonine kinase (AKT) activation in 3D DP spheroids. This was correlated with an increase in the diameter of the 3D DP spheroids. The activation of the wingless and INT-1 (Wnt)/ $\beta$-catenin signaling pathway, which is associated with hair growth induction in the DP, was evaluated using the T cell-specific transcription factor and lymphoid enhancer-binding factor (TCF/LEF) transcription factor reporter assay; results indicated significantly increased luciferase activity. In addition, we were able to demonstrate increased expression of the target genes, WNT5 a and LEF1, using quantitative real-time PCR assay. Lastly, significantly elevated expression of signature genes associated with hair induction was demonstrated in the 3D DP spheroids treated with silibinin. These results suggest that silibinin promotes proliferation and hair induction through the AKT and Wnt/ $\beta$-catenin signaling pathways in 3D DP spheroids. Silibinin can be a potential candidate to promote hair proliferation.

Keywords: Silibinin, dermal papilla cells, Akt, hair growth inductive, spheroid culture

\section{Introduction}

The flavonoid silibinin is a major component of silymarin, a mixture of flavonoids that comprise a subset of the secondary metabolites derived from Silybum marianum [1]. A number of recent in vivo studies have indicated that silibinin is a strong antioxidant with effective pharmacological and chemopreventive properties that can lower the risk of various cancers including those of the skin, prostate, and lungs [2-4]. Silibinin has also been shown to neutralize the dermatological toxicity induced by various chemicals and ultraviolet-B radiation [1]. Silymarin and silibinin have been found to inhibit TPA (12-O-tetradecanoyl13 phorbol acetate) and okadaic acid-induced carcinogenesis in skin [5, 6]. Studies in mouse models have revealed that silymarin decreases TPA-induced skin edema and epidermal hyperplasia [5]. Additionally, silibinin has been shown to function as an anti-inflammatory agent, specifically, by preventing both the activation of oxidant-induced cyclooxygenase and production of TPA-induced cytokines, including tumor necrosis factor alpha (TNF- $\alpha$ ) and interleukin 1 alpha (IL-1 $\alpha$ ) in mouse skin [5]. Studies in mice have revealed that silibinin protects skin from sunburn, hyperplasia, and cell death induced by exposure to ultraviolet B (UVB) [5]. Based on these results, silibinin has been used in cosmetic products to prevent cutaneous oxidative damage and photoaging [5]. Although numerous studies have revealed the efficacy of silibinin-based 
pharmacological and dermatological treatments, there has been no attempt to investigate the effect of silibinin on hair induction. The current study is the first to reveal that silibinin has hair growth-promoting properties in 3Dcultured spheroid, human dermal papilla (DP) cells.

Scalp hair, composed mainly of keratin, serves to protect from harmful elements and, notably, also contributes to one's personality [7]. One of the most prominent features of hair is the specific growth pattern consisting of three distinct phases-anagen, catagen, and telogen [7, 8]. Hair growth and new hair induction are therefore tightly regulated by the hair cycle [7]. Exposure of hair to internal and external factors-hormones, stress, pollution, and other factors that are undefined-results in a change in and shortening of the normal hair-growth cycle [7]. This change is linked to the shortening of the anagen phase, an increase in the proportion of hairs in catagen, and the lengthening of telogen resulting in hair loss, or alopecia $[7,8]$. The hair cycle is regulated mainly by small specialized organs-hair follicles. Hair follicles are located at the root of the hair within the skin [9]. One of the most important components of the hair follicle is a specialized population of mesenchymal cells called the dermal papilla (DP) [9]. The DP provides important regulatory signals to the hair follicle; therefore, it has a pivotal role in hair induction, growth, and cycling [8]. In alopecia patients, the hair follicle becomes miniaturized, and this can lead to decreased induction of hair growth by the DP [10]. A number of studies focused on elucidating the mechanisms of hair loss and identifying therapeutic candidates have been conducted using mouse models with hair loss phenotypes as well as human DP cells. Mouse skin is different, however, from human skin with respect to the hair cycle, skin function, stem cell activity, and hormonal dependence [11]. It has also been reported that mice do not suffer from androgenetic alopecia, which is the most common type of hair loss disease in humans [12]. Due to these limitations, it is preferable to use human DP cells; however, it is known that the common monolayer-based animal cell culture (2D) system does not support the potential of DP cells to induce hair growth [13]. Recent research has resulted in the development of the $3 \mathrm{D}$ culture system to enable formation of DP spheroids that have in vivo, DP-like morphology [13]. This culture system has been accepted for hair induction studies because the induction of hair growth is reproducible. The 3D DP spheroids have also been shown to induce hair growth de novo when transplanted [14, 15]. At the molecular level these results can be correlated with the expression of DP signature genes, including alkaline phosphatase $(A L P L)$, versican (VCAN), fibroblast growth factor 7 (FGF7), and bone morphogenetic protein 2 (BMP2). DP cells are known to provide signals including FGF7 and BMP2 that stimulate initiation of the anagen phase in hair follicles [16]. ALPL is a DP-specific marker that maintains the hair-inductive function [17]. VCAN, additionally, is a component of the extracellular matrix that plays a role in hair follicle formation [17]. The DP-specific Wnt/ $\beta$-catenin signaling has been found to play an important role in anagen hair growth and new hair induction [18].

In this study, we have demonstrated the effect of silibinin on hair growth induction in 3D DP spheroids. Silibinin induces AKT-dependent gain of cell viability; this result was correlated with increase in the size of DP spheroids. Using luciferase and qRT-PCR assays, we have revealed that silibinin activates the Wnt/ $\beta$-catenin signaling pathway in DP spheroids in an AKT-dependent manner. Additionally, we have observed that silibinin treatment significantly increases expression levels of DP signature genes. Therefore, our results indicate that silibinin may be a therapeutic candidate for hair loss treatment.

\section{Materials and Methods}

\section{Cell Culture, Plasmids, and Reagents}

The human hair follicle DP cells were cultured in $5 \% \mathrm{CO}_{2}$ at $37^{\circ} \mathrm{C}$ in mesenchymal stem cell medium (MSCM; Innoprot) supplemented with $5 \%(\mathrm{v} / \mathrm{v})$ fetal bovine serum (FBS) according to the instructions provided by Innoprot (Bizakaia, Spain). TCF/ LEF luciferase reporter plasmids were obtained from Promega (USA). A selective phosphatidylinositol 3-kinase (PI3K) inhibitor, LY294002, and silibinin were purchased from Sigma-Aldrich (USA).

Three-dimensional (3D) culture of DP cells. Three-dimensional (3D) culture of DP cells was performed as previously described [19]. Briefly, cells were maintained in 2D culture as described above. To obtain one spherical structure of the cells, cultured human DP cells $\left(5 \times 10^{4}\right.$ cells/well) were seeded into a 96-well, clear, round-bottom ultra-low attachment microplate (Corning Incorporated). After the formation of the unified spheres, the spheres were treated with silibinin for $48 \mathrm{~h}$. Finally, the diameters of the spheres were measured by phase-contrast microscopy.

\section{Cell Viability Analysis}

Cell viability was determined as described in the supplier's protocol of WST-1 assay (EZ-Cytox cell viability assay kit; ITSBio, Korea). Briefly, $1 \times 10^{4}$ cells were seeded per well in 96-well plates and maintained in complete medium for $24 \mathrm{~h}$. After incubation, the cells were treated with the indicated doses of silibinin and 
incubated for a further $48 \mathrm{~h}$. WST-1 solution was subsequently added to each well and allowed to remain for $0.5 \mathrm{~h}$ to allow formazan formation to occur. Cell viability was determined by measuring absorbance at $450 \mathrm{~nm}$ using an iMark microplate reader (Bio-Rad Laboratories, USA).

\section{Luciferase Reporter Assay}

The TCF/LEF1 promoter-carrying luciferase reporter plasmid was co-transfected with the $\mathrm{pSV}$ - $\beta$-galactosidase (pSV- $\beta$-gal) plasmid using Lipofectamine 3000 (Invitrogen). pSV- $\beta$-gal plasmid was used as a control for transfection efficiency. Twenty-four hours after transfection, the cells were grown by the 3D culture method and were subsequently treated with silibinin alone or in combination with LY294002 (Sigma, USA). After $24 \mathrm{~h}$ of treatment, the cells were lysed using passive lysis buffer (Promega) and the lysates were incubated with D-luciferin (Sigma-Aldrich) to determine luciferase activity, which was then measured using a Glomax 96 Microplate Luminometer (Turner BioSystems, USA). $\beta$-gal activity was analyzed using the Luminescent $\beta$-galactosidase Detection Kit II (Clontech Laboratories Inc., USA). Relative luciferase activity was determined by normalizing the levels to $\beta$-gal activity.

\section{Western Blotting}

Total cell lysates were prepared, and protein extracts were obtained after lysis for $30 \mathrm{~min}$ at $4^{\circ} \mathrm{C}$ in RIPA buffer $(50 \mathrm{mM}$ Tris$\mathrm{HCl}, \mathrm{pH}$ 7.4, $150 \mathrm{mM} \mathrm{NaCl}, 1 \% \mathrm{NP}-40,0.5 \%$ Deoxycholic acid, $0.1 \%$ SDS). Protein lysates were fractionated by sodium dodecyl sulfate/polyacrylamide gel electrophoresis (SDS-PAGE) followed by immunoblotting. The primary antibodies used for immunoblotting analysis were as follows: antibodies against NCAM (ab75813) and ALPL (ab108337) purchased from Abcam (UK); antibodies targeting AKT (5G3), p-AKT (S473), and $\beta$-catenin (9562) from Cell Signaling Technology (USA); and the antibody against $\beta$-actin (a5441) from Sigma-Aldrich. Following incubation with the primary antibodies, the membranes were incubated with goat anti-mouse or goat antirabbit horseradish peroxidase-conjugated secondary antibodies (Cell Signaling Technology, USA). The stained bands were visualized by using the Pierce ECL western blotting substrate (Thermo Scientific, USA). $\beta$-actin was used as a loading control.

\section{Quantitative RT-PCR (qRT-PCR) Analysis}

Total RNA was extracted from human DP spheres using TRIzol reagent (Invitrogen; Thermo Fisher Scientific). cDNA was synthesized from $2 \mu \mathrm{g}$ of total RNA using M-MLV reverse transcriptase (Invitrogen). Quantitative RT-PCR (qRT-PCR) was carried out using a HOT FIREPol EvaGreen qPCR Mix Plus (Solis BioDyne, Tartu, Estonia) with a Step OnePlus Real-Time PCR System (Applied Biosystems-Thermo Fisher Scientific, USA) according to the manufacturer's protocol. The primers used for amplification of specific genes were as follows: Wnt family member 5A (WNT5A), 5'-TCCACCTTCCTCTTCACACTGA-3' (forward) and 5'-CGT GGCCAGCATCACATC)-3' (reverse); human alkaline phosphatase (ALPL), 5'-ATTGACCACGGGCACCAT-3' (forward) and 5'-
CTCCACCGCCTCATGCA-3' (reverse); versican (VCAN), 5'GGCAATCTATTTACCAGGACCTGAT-3' (forward) and 5'-TGG CACACAGGTGCATACGT-3' (reverse); bone morphogenetic protein 2 (BMP2), 5'-GAGGTCCTGAGCGAGTTCGA-3' (forward) and 5'-TCTCTGTTTCAGGCCGAACA-3' (reverse); fibroblast growth factor 7 (FGF7), 5'- ATCAGGACAGTGGCAGTTGGA-3' (forward) and 5'-AACATTTCCCCTCCGTTGTGT-3' (reverse); enhancer-binding factor-1 (LEF1), 5'-AATGAGAGCGAATGT CGTTGC-3' (forward) and 5'-GCTGTCTTTTTTCCGTGCTA-3' (reverse). Each mRNA expression level was calculated using the $2^{-\Delta A C t}$ method and was normalized to the expression level of the $\beta$-actin housekeeping gene. The following primers were used to amplify the human $\beta$-actin gene: 5 '-GGATTCCTATGTGGG CGACGA-3' (forward) and 5'-CGCTCGGTGAGGATCTTCATG-3' (reverse).

\section{Statistical Analysis}

All data are presented as mean \pm standard deviation (SD), and normally distributed data have been evaluated using a two-tailed Student's $t$-test as indicated in the figure legends. All analyses were performed with triplicate independent experiments. Results are considered statistically significant when $\underline{x}<0.05$.

\section{Results}

\section{Evaluation of Cytotoxic Activity of Silibinin in 2D- and 3D-Cultured Human DP Cells}

Before the evaluation of silibinin as an agent of hair induction in human DP cells, we sought to investigate its potential cytotoxicity and overall effect on viability in these cells (Fig. 1A). First, human DP cells were cultured in a monolayer followed by treatment with the various doses $(0$ to $200 \mu \mathrm{M}$ ) of silibinin for $24 \mathrm{~h}$. As shown in Fig. 1B (closed circle), silibinin did not induce a cytotoxic effect on the 2Dcultured human DP cells treated with doses less than $20 \mu \mathrm{M}$; however, treatment with higher doses, specifically, $50 \mu \mathrm{M}, 100 \mu \mathrm{M}$, or $200 \mu \mathrm{M}$ of silibinin resulted in a decrease in cell viability by $12.49 \%, 26.86 \%$, and $46.69 \%$, respectively. The decrease in viability was significant compared to that observed with control (DMSO-treated) cells. Noteworthy was an increase of $13.53 \%$ in the viability of cells treated with $10 \mu \mathrm{M}$ silibinin compared to that observed in cells treated as control. Next, we investigated the effect of silibinin on viability in 3D-spheroid-cultured, human DP cells. To form DP spheres, cells were seeded and incubated to be grown in hydrogel-coated, low attachment round surfaces as described in Materials and Methods. Unlike the results with 2D-cultured DP cells, we found that treatment of 3D-spheroid-cultured, human DP cells with lower doses-less than $20 \mu \mathrm{M}$-of silibinin significantly increased cell viability in a dose-dependent manner (Fig. 1B; open 
A<smiles>COc1cc(C2Oc3ccc(C4Oc5cc(O)cc(O)c5C(=O)C4O)cc3OC2CO)ccc1O</smiles>

Silibinin

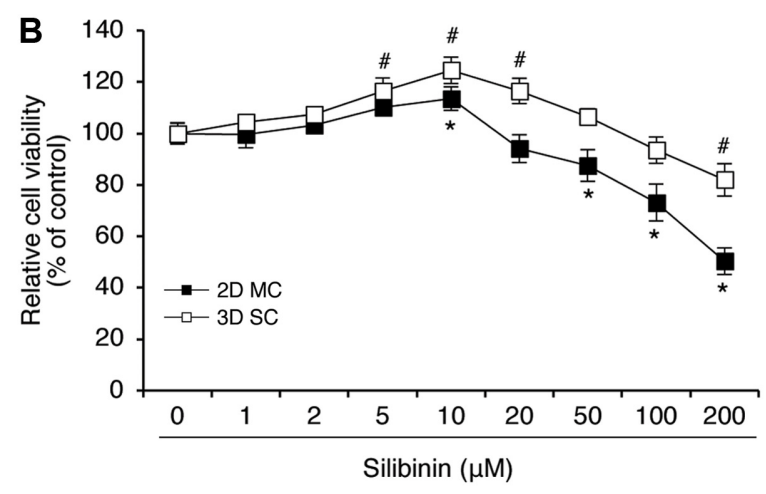

Fig. 1. Effect of silibinin on the viability of DP cells.

(A) Chemical structure of silibinin. (B) Monolayered (2D)- and 3D-cultured DP cells were treated with different concentrations of silibinin (1$200 \mu \mathrm{M})$ for $24 \mathrm{~h}$ and cell viability was determined using the WST-1 assay. The results are expressed as percent viable cells relative to the control. The data are presented as the mean \pm S.D. of three independent experiments. Values marked by an * and ${ }^{*}$ were considered statistically significant with $p<0.05$. 2D, two-dimensional; 3D, three-dimensional.

circle). A significant cytotoxic effect was observable only in cells treated with the highest dose of silibinin (Fig. 1B; open circle). Overall, these results suggest that silibinin has a viability-promoting effect in 3D-spheroid-cultured, human DP cells.

\section{Treatment of Silibinin Induces Akt Activation in 3D DP Spheroids}

The serine/threonine kinase Akt, also known as protein kinase $\mathrm{B}(\mathrm{PKB})$, has a pivotal role in promoting cell growth in various cells, and the activity of Akt is tightly regulated by phosphorylation [20]. We have, considering the function of Akt, performed experiments to determine if the viability-increasing effect of silibinin might be mediated by Akt activation in 3D-spheroid-cultured, human DP cells. Cells were incubated to be self-assembled for sphere formation and treated with 0 to $10 \mu \mathrm{M}$ silibinin for $24 \mathrm{~h}$. Akt protein levels and phosphorylation status were analyzed using immunoblotting assays with specific antibodies. As expected, silibinin was found to increase the level of Akt
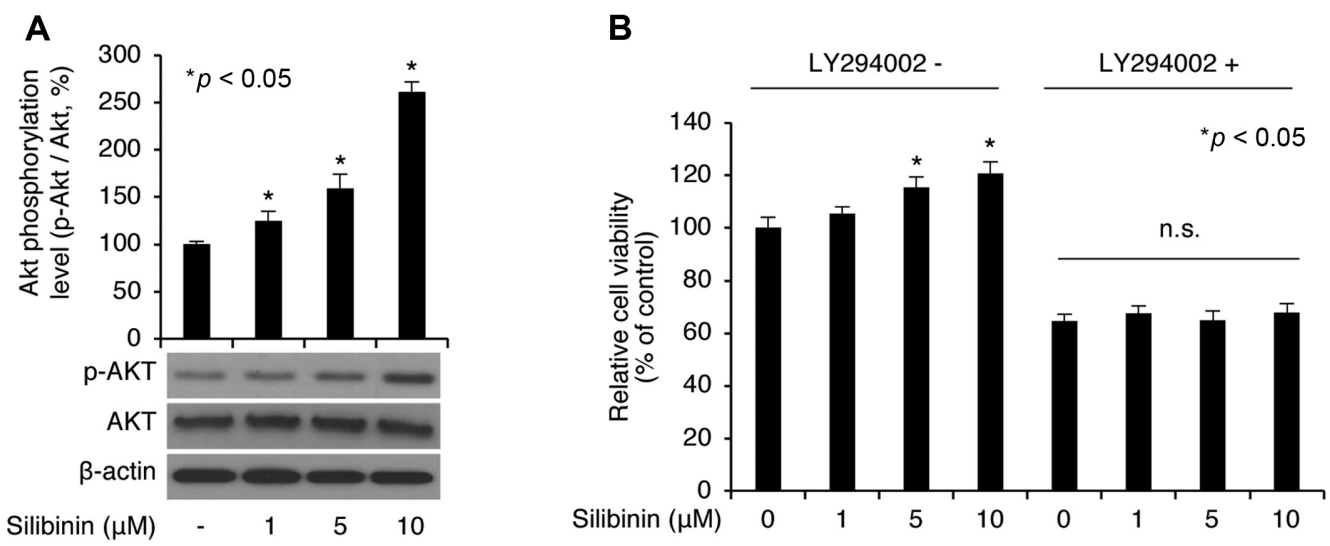

Fig. 2. Effect of AKT activation on the viability in silibinin-treated 3D DP cells.

(A) The effect of silibinin on AKT phosphorylation/activation in 3D-cultured DP cells. DP cells were grown in a 3D-cultured system, treated with different concentrations of silibinin $(1-10 \mu \mathrm{M})$ for $24 \mathrm{~h}$, and cell viability was determined through immunoblotting assay with specific antibodies. Quantification of p-Akt protein was carried out using the Image-J program and normalized to total Akt protein levels. (B) AKT-dependent regulation of cell viability in silibinin-treated 3D DP cells. DP cells were grown in a 3D-cultured system and co-treated with different concentrations of silibinin and LY294002 for $24 \mathrm{~h}$. Cell viability was determined through WST-1 assay. The results are expressed as percent cell viability relative to the control. The data are presented as the mean \pm S.D. of three independent experiments. Values indicated by * were considered statistically significant with $p<0.05$. 
A

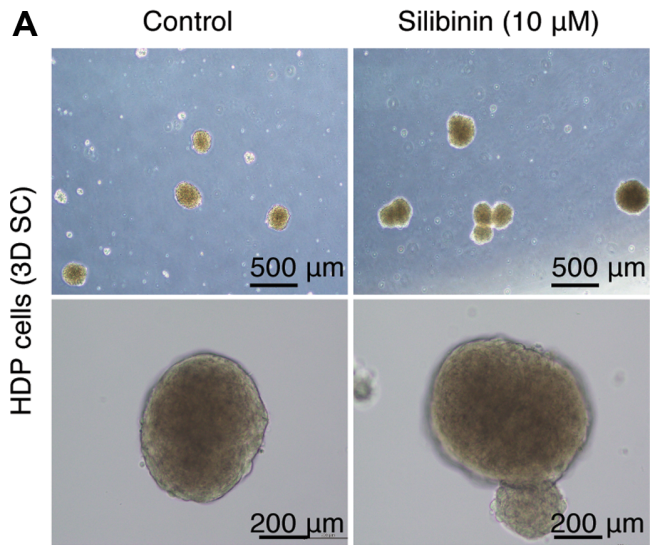

B

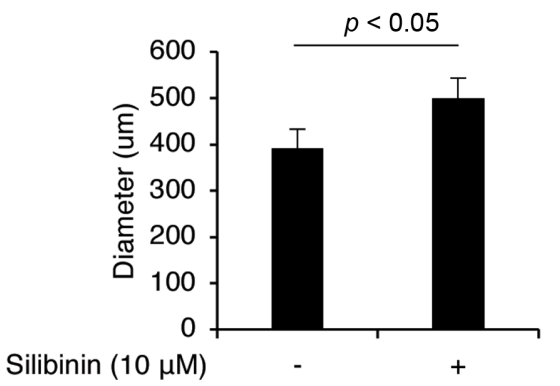

Fig. 3. Effect of silibinin on the spheroid formation of DP cells. (A) Human DP (HDP) cells were grown in a 3D-cultured system, and then treated with the indicated concentrations of silibinin $(0$ or $10 \mu \mathrm{M})$ for $24 \mathrm{~h}$, and phase-contrast images of spheroids captured. (B) The spheroid diameter was quantified and graphed. The data are presented as the mean \pm S.D. of three independent experiments. Values of $p<0.05$ were considered statistically significant. 3D SC, three-dimensional spheroid cultured.

A

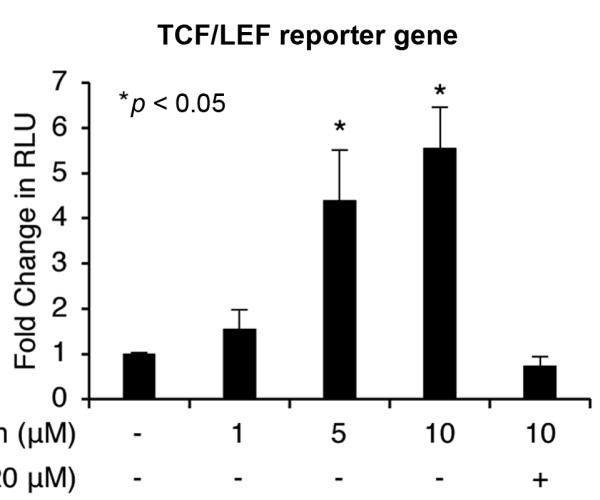

phosphorylation in a dose-dependent manner (Fig. 2A). Next, we investigated the role of Akt phosphorylation in the viability-promoting effect of silibinin in human DP cells. We confirmed that silibinin was able to increase the viability of 3D-spheroid-cultured, human DP cells in an Akt-specific manner; the increase in viability was not detectable in controls treated with LY294002, a specific inhibitor of the PI3K-mediated Akt activation (Fig. 2B). These results are indicative that silibinin promotes viability through Akt activation in 3D-spheroid-cultured, human DP cells.

\section{Treatment with Silibinin Increases Sphere Formation in Human DP Cells}

Recent reports have revealed that the ability of DP cells to form spheroids is connected to their potential to induce hair growth [15]. We therefore carried out experiments to investigate whether the influence of silibinin on the viability of 3D-spheroid-cultured, human DP cells can promote DP sphere formation. Human DP cells were grown in the 3D culture system followed by treatment with silibinin for $24 \mathrm{~h}$. The size of the DP spheroids was analyzed and quantified using a microscopy-based image acquisition and sphere diameter analysis. As shown in Fig. 3A, silibinin treatment was found to alter the spheroid size of the DP cells. The silibinin treatment-induced increase in the size of DP spheres was notably significant compared to that seen in DMSO-treated DP cells (Fig. 3B). These results suggest that silibinin enhances the formation of DP spheroids in vitro.

B

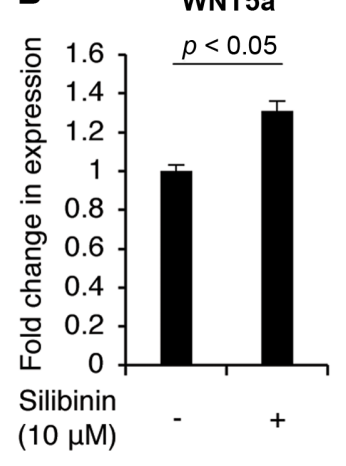

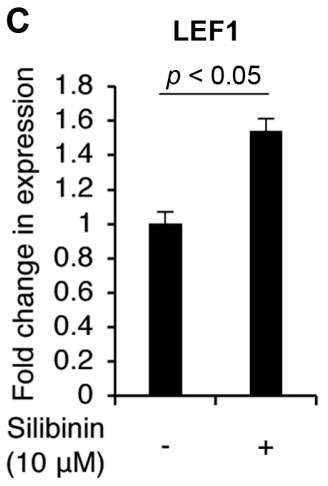

Fig. 4. Effect of silibinin on the activity of Wnt/ $\beta$-catenin signaling on 3D DP spheroids.

(A) The effect of silibinin on the luciferase activity of TCF/LEF reporter plasmid in 3D DP spheroids. DP cells were transfected with the reporter and $\beta$-galactosidase plasmids. Cells were then grown in a 3D-cultured system followed by treatment with silibinin for $24 \mathrm{~h}$. The luciferase activity was determined by normalizing the $\beta$-galactosidase activity. (B and $\mathbf{C}$ ) The effect of silibinin on the expression levels of target genes of Wnt/ $\beta$ catenin signaling, WNT5A (B) and LEF1 (C) in 3D DP spheroids. DP cells were grown in a 3D-cultured system, treated with the indicated concentrations of silibinin $(0$ or $10 \mu \mathrm{M})$ for $24 \mathrm{~h}$, and then the mRNA levels of these genes were assessed by qRT-PCR analysis. The data are presented as the mean \pm S.D. of three independent experiments. Values of $p<0.05$ were considered statistically significant. 


\section{Treatment of Silibinin Activates Wnt $/ \beta$-Catenin Signaling in 3D DP Spheroids}

The hair-inductive property of DP cells is characterized by activation of the Wnt/ $\beta$-catenin signaling pathways at the molecular level [18]. Based on this knowledge, we have carried out experiments to examine whether silibinintreated DP spheroids display activation of the Wnt/ $\beta$ catenin signaling pathway. DP cells were first transfected with the luciferase reporter plasmids containing the TCF/ LEF responsive elements controlled by $\beta$-catenin and then grown in the 3D culture system followed by treatment with silibinin for $24 \mathrm{~h}$. As shown in Fig. 4A, the luciferase activity was found to increase in a dose-dependent manner in 3D DP spheroids treated with silibinin. This effect was, however, nullified by LY294002 treatment. To further confirm the luciferase data, we analyzed expression levels of downstream targets genes (WNT5a and LEF1) of the $\mathrm{TCF} / \mathrm{LEF}$ transcription factor. qRT-PCR analysis of mRNA levels revealed increased expression of target genes in silibinin-treated 3D DP spheroids than in control spheroids. Overall, these results indicate that silibinin activates the Wnt/ $\beta$-catenin signaling pathway in 3D DP spheroids.

\section{Treatment with Silibinin Upregulates DP Signature Genes in 3D DP Spheroids}

We next examined whether silibinin can influence expression of DP signature genes that are important regulators of in vivo DP inductivity [18]. DP cells were grown in the 3D culture system followed by treatment with silibinin for $24 \mathrm{~h}$. Using a qRT-PCR assay, we determined the expression levels of DP signature genes, including
ALPL, VCAN, FGF7, and BMP2. As shown in Fig. 5A, silibinin treatment resulted in an increase in the levels of DP signature transcripts in 3D DP spheroids. To verify these data at the level of the protein, immunoblotting was performed using specific antibodies. We were able to demonstrate that the levels of these proteins were also increased by silibinin treatment of 3D DP spheroids (Fig. 5B). The elevated levels of $\beta$-catenin protein revealed by this experiment offer another line of evidence for the activation of the $\mathrm{Wnt} / \beta$-catenin signaling pathway in silibinin-treated 3D DP spheroids (Fig. 5B). Taken together, our results indicate that silibinin treatment can enhance the hair-inductive property of 3D DP spheroids by activating the Wnt/ $\beta$-catenin signaling pathway.

\section{Discussion}

In this study, we have evaluated the potential effect of silibinin on hair growth induction in 3D-cultured DP cells. Studies have indicated that hair loss is a common sign of aging and, importantly, has negative psychosocial effects because of its association with an individual's pursuit of beauty and self-esteem [21]. Therefore, it is important not only to understand the etiologic factors and molecular mechanisms behind hair loss, but also to develop natural candidates for the treatment of this condition. Oxidative stress is an important factor that contributes to the aging of skin and related dermatological conditions. The knowledge that silibinin acts as a scavenger of reactive oxygen species (ROS) has led to its widespread dermatologic applications for the prevention of aging in skin [1]. The DP cells of
A

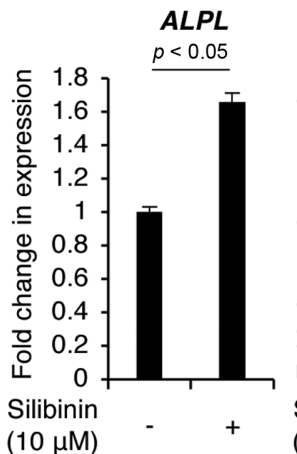

$(10 \mu \mathrm{M})$

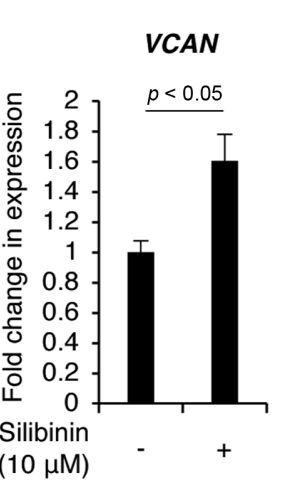

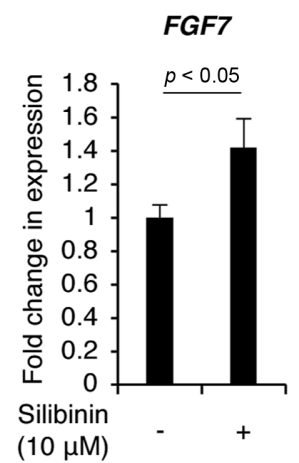

B

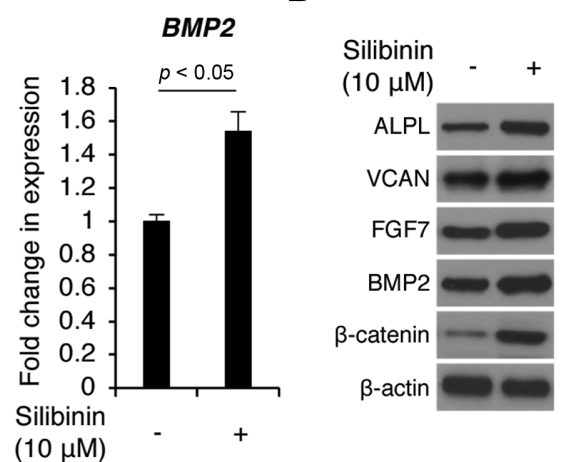

Fig. 5. Effect of silibinin on the expression of DP signature genes in 3D DP spheroids.

(A) DP cells were grown in a 3D-cultured system, treated with the indicated concentrations of silibinin $(10 \mu \mathrm{M})$ for $24 \mathrm{~h}$, and then the mRNA levels of the genes ALPL, VCAN, FGF7, and BMP2 were assessed by qRT-PCR analysis using specific primer sets. The data are presented as the mean \pm S.D. of three independent experiments. Values represented by an * were statistically significant with $p<0.05$. (B) Changes in the protein levels of $A L P L, V C A N, F G F 7, B M P 2$, and $\beta$-catenin were determined by immunoblot analysis using specific antibodies. 
alopecia patients have been shown to display elevated levels of ROS, and this has been proposed to contribute to the etiology of hair loss [22]. Despite these cues, there has been no study so far to evaluate the potential role of silibinin in the induction of hair growth. In this study, we have addressed this gap-in-knowledge using the 3D spheroid DP culture model.

The turnover of hair follicles occurs over a course of a cycle that includes the growth and proliferation (anagen), involution (catagen) and resting (telogen) phases; shortening of the anagen phase is a common sign observed in alopecia [23]. Therefore, studies for developing new therapies and identifying potential agents to inhibit hair loss have focused on the effect of hair follicle DP proliferation, as commonly measured by a cell viability assay $[23,24]$. AKT is a serine/threonine kinase, and its activation and phosphorylation levels are tightly linked to the proliferation of various cells [20]. Studies have also indicated that AKT promotes dermal papilla cell survival. Additionally, studies analyzing various anti-alopecia candidates have demonstrated the hair growth-promoting effects of AKT activation in DP cells [23, 25]. Our studies reveal that there is a dose-dependent increase in the cell viability and the p-AKT levels with the total AKT protein level remaining unchanged; this is indicative that silibinin induces AKT activation in 3D-cultured DP cells.

As mentioned above, 2D-cultured DP cells lose their ability to induce hair growth; however, three dimensionalculture conditions can make DP cells aggregate to form spheroids that are morphologically similar to intact papillae [13]. These results were suggestive that 3D-cultured DP spheroids could induce hair growth; other studies also proved that the potential for hair growth induction is partially recovered in the 3D DP spheroids and that these spheroids can induce new hair growth $[14,26]$. We found that the diameter of 3D DP spheroids increased after treatment with silibinin. Androgenic alopecia involves miniaturization of the hair follicle with reduction in the follicle diameter, and shortening of the hair shaft [27, 28]. Our results suggest that the silibinin-mediated enhancement of DP spheroid formation could indeed be related to its hair growth induction potential $[14,26]$.

The Wnt/ $\beta$-catenin signaling pathway is tightly linked to the maintenance of the anagen phase, and the hair-inductive property of DP cells [29]. When this pathway is activated, stabilized $\beta$-catenin is translocated into the nucleus where it interacts with the TCF/LEF transcription factor to activate transcription of downstream target genes, including WNT5A and LEF1 [30]. The TCF/LEF luciferase reporter assay was, therefore, used to evaluate Wnt/ $\beta$-catenin signaling activity; we found that the luciferase activity was significantly increased in 3D DP spheroids after silibinin treatment than in the control-treated cells. However, a controversial result has been reported regarding the role of silibinin as an inhibitor of Wnt/ $\beta$-catenin signaling pathway [31]. These inconsistent results may be accounted for by differences in target cells and treatment dosages. The inhibitory effect of silibinin on Wnt/ $\beta$-catenin signaling was demonstrated in prostate and breast cancer cells, not in normal or DP cells [31]. Additionally, the treatment doses $(50,100$, and $200 \mu \mathrm{g} / \mathrm{ml})$ of silibinin used were markedly higher than the dose $(10 \mu \mathrm{g} / \mathrm{ml})$ used in this study [31]. To confirm our results, qRT-PCR was performed to assess the expression levels of the target genes-WNT5A and LEF1of the Wnt/ $\beta$-catenin signaling pathway; we found that the expression of these genes was significantly elevated in $3 \mathrm{D}$ DP spheroids after silibinin treatment than in the control, indicating that silibinin activates $\mathrm{Wnt} / \beta$-catenin signaling pathway in 3D DP spheroids. Similar results were also shown in the other report, in which silybin (the other name of silibinin) synergizes with Wnt3a in activation of the Wnt/ $\beta$-catenin signaling pathway [32]. Specifically, the role of silibinin as an activator of the Wnt/ $\beta$-catenin signaling pathway was shown in 3T3-L1 fibroblasts treated with the lower doses $(2.5-10 \mu \mathrm{g} / \mathrm{ml})$ of silibinin. Lastly, we found significantly increased expression of signature genes in 3D DP spheroids treated with silibinin. These results indicate that silibinin could be a candidate for hair loss treatment via its action to promote cell viability, sphere formation, and Wnt/ $\beta$-catenin signaling in DP cells. Further in-depth studies of the Wnt/ $\beta$-catenin signaling pathway and clinical evaluation are, however, required to better understand the effect of silibinin treatment on hair induction.

Minoxidil and finasteride are the currently available drugs for hair loss prevention [33]. Minoxidil acts as an anti-androgen by inhibiting androgen-mediated activation of the $5 \alpha$-reductase enzyme that converts testosterone into $5 \alpha$-dehydrotestosterone ( $5 \alpha$-DHT), and finasteride has an inhibitory effect on the conversion of testosterone to $5 \alpha$ DHT [33]. These drugs have been regarded as the primary pharmacologic therapies for hairloss, especially androgenetic alopecia [9]. It is generally accepted that increased androgen activity leads to hair follicle miniaturization; in addition, the androgen-mediated overproduction of $5 \alpha$-DHT leads to shortening of the anagen phase in the DP and consequent downregulation of hair production [9]. Our data suggest that silibinin can potentially be used for hair loss treatment by activating hair growth in DP cells. This approach is 
different from the mechanisms of the developed and currently available drugs including minoxidil and finasteride for prevention of further hair loss. Studies have indicated that approaches using drugs to target pathways come with several limitations. The clinical effectiveness of minoxidil and finasteride has remained low with response rates of $35 \%$ and $48 \%$, respectively. Unwanted side effects including irritation, itchiness, and abnormal ejaculation and sexual function have also been reported [33-35]. These limitations have encouraged the development of alternative treatment strategies; one such strategy is focused on the promotion of hair growth rather than the prevention of further hair loss $[19,36]$, and the other strategy relies on the effectiveness of extracts or specific agents derived from microorganisms or plants on the induction of hair growth [23, 37, 38]. Our study with silibinin is consistent with these alternative approaches outlined above.

In summary, silibinin treatment enhances spheroid formation of DP cells through the activation of AKT and Wnt $/ \beta$-catenin signaling pathways. Our data suggest that silibinin may have therapeutic potential for the treatment of hair loss by promoting hair growth induction in the DP.

\section{Acknowledgment}

This work was supported by Konkuk University Medical Center Research Grant 2016.

\section{Conflict of Interest}

The authors have no financial conflicts of interest to declare.

\section{References}

1. Singh RP, Agarwal R. 2009. Cosmeceuticals and silibinin. Clin. Dermatol. 27: 479-484.

2. Singh RP, Agarwal R. 2002. Flavonoid antioxidant silymarin and skin cancer. Antioxid. Redox Signal. 4: 655-663.

3. Singh RP, Dhanalakshmi S, Tyagi AK, Chan DC, Agarwal C, Agarwal R. 2002. Dietary feeding of silibinin inhibits advance human prostate carcinoma growth in athymic nude mice and increases plasma insulin-like growth factorbinding protein-3 levels. Cancer Res. 62: 3063-3069.

4. Singh RP, Deep G, Chittezhath M, Kaur M, Dwyer-Nield LD, Malkinson AM, et al. 2006. Effect of silibinin on the growth and progression of primary lung tumors in mice. J. Natl. Cancer Inst. 98: 846-855.

5. Lahiri-Chatterjee M, Katiyar SK, Mohan RR, Agarwal R. 1999. A flavonoid antioxidant, silymarin, affords exceptionally high protection against tumor promotion in the SENCAR mouse skin tumorigenesis model. Cancer Res. 59: 622-632.

6. Zi X, Mukhtar H, Agarwal R. 1997. Novel cancer chemopreventive effects of a flavonoid antioxidant silymarin: inhibition of mRNA expression of an endogenous tumor promoter TNF? Biochem. Biophys. Res. Commun. 239: 334-339.

7. Choi B. 2018. Hair-growth potential of ginseng and its major metabolites: a review on its molecular mechanisms. Int. J. Mol. Sci. 19: 2703.

8. Alonso L, Fuchs E. 2006. The hair cycle. J. Cell Sci. 119: 391393.

9. Westgate GE, Botchkareva NV, Tobin DJ. 2013. The biology of hair diversity. Int. J. Cosmetic Sci. 35: 329-336.

10. Santos Z, Avci P, Hamblin MR. 2015. Drug discovery for alopecia: gone today, hair tomorrow. Expert Opin. Drug Discov. 10: 269-292.

11. Panteleyev AA. 2016. Putting the human hair follicle cycle on the map. J. Invest. Dermatol. 136: 4-6.

12. Porter RM. 2003. Mouse models for human hair loss disorders. J. Anat. 202: 125-131.

13. Topouzi H, Logan NJ, Williams G, Higgins CA. 2017. Methods for the isolation and 3D culture of dermal papilla cells from human hair follicles. Exp. Dermatol. 26: 491-496.

14. Higgins CA, Chen JC, Cerise JE, Jahoda CAB, Christiano AM. 2013. Microenvironmental reprogramming by threedimensional culture enables dermal papilla cells to induce de novo human hair-follicle growth. Proc. Natl. Acad. Sci. USA. 110: 19679-19688.

15. Higgins CA, Richardson GD, Ferdinando D, Westgate GE, Jahoda CAB. 2010. Modelling the hair follicle dermal papilla using spheroid cell cultures. Exp. Dermatol. 19: 546-548.

16. Greco V, Chen T, Rendl M, Schober M, Pasolli HA, Stokes N, et al. 2009. A two-step mechanism for stem cell activation during hair regeneration. Cell Stem Cell. 4: 155-169.

17. Yang C-C, Cotsarelis G. 2010. Review of hair follicle dermal cells. J. Dermatol. Sci. 57: 2-11.

18. Zhou L, Yang K, Xu M, Andl T, Millar SE, Boyce S, et al. 2016. Activating $\beta$-catenin signaling in CD133-positive dermal papilla cells increases hair inductivity. FEBS J. 283: 2823-2835.

19. Choi YM, An S, Lee J, Lee JH, Lee JN, Kim YS, et al. 2017. Titrated extract of Centella asiatica increases hair inductive property through inhibition of STAT signaling pathway in three-dimensional spheroid cultured human dermal papilla cells. Biosci. Biotechnol. Biochem. 81: 2323-2329.

20. Manning BD, Toker A. 2017. AKT/PKB signaling: navigating the network. Cell 169: 381-405.

21. Alfonso M, Richter-Appelt H, Tosti A, Viera MS, Garcia M. 2005. The psychosocial impact of hair loss among men: a multinational European study. Curr. Med. Res. Opin. 21: 1829-1836.

22. Upton JH, Hannen RF, Bahta AW, Farjo N, Farjo B, Philpott MP. 2015. Oxidative stress-associated senescence in dermal 
papilla cells of men with androgenetic alopecia. J. Invest. Dermatol. 135: 1244-1252.

23. Rastegar H, Ashtiani HA, Aghaei M, Barikbin B, Ehsani A. 2015. Herbal extracts induce dermal papilla cell proliferation of human hair follicles. Ann. Dermatol. 27:667-675.

24. Rho S, Park S, Hwang S, Lee M, Kim C, Lee I, et al. 2005. The hair growth promoting effect of extract and its molecular regulation. J. Dermatol. Sci. 38: 89-97.

25. Woo H, Lee S, Kim S, Park D, Jung E. 2017. Effect of sinapic acid on hair growth promoting in human hair follicle dermal papilla cells via Akt activation. Arch. Dermatol. Res. 309: 381-388.

26. Kang BM, Kwack MH, Kim MK, Kim JC, Sung YK. 2012. Sphere formation increases the ability of cultured human dermal papilla cells to induce hair follicles from mouse epidermal cells in a reconstitution assay. J. Invest. Dermatol. 132: 237-239.

27. de Lacharriere $O$, Deloche $C$, Misciali C, Piraccini BM, Vincenzi C, Bastien P, et al. 2001. Hair diameter diversity: a clinical sign reflecting the follicle miniaturization. Arch. Dermatol. 137: 641-646.

28. Whiting DA. 2001. Possible mechanisms of miniaturization during androgenetic alopecia or pattern hair loss. J. Am. Acad. Dermatol. 45: S81-86.

29. Kishimoto J, Burgeson RE, Morgan BA. 2000. Wnt signaling maintains the hair-inducing activity of the dermal papilla. Genes Dev. 14: 1181-1185.

30. Zhou D, Tan RJ, Fu H, Liu Y. 2015. Wnt/ $\beta$-catenin signaling in kidney injury and repair: a double-edged sword. Lab. Invest. 96: 156-167.
31. Lu W, Lin C, King TD, Chen H, Reynolds RC, Li Y. 2012. Silibinin inhibits $W n t / \beta$-catenin signaling by suppressing Wnt co-receptor LRP6 expression in human prostate and breast cancer cells. Cell. Signal. 24: 2291-2296.

32. Kim T, Oh S. 2012. Silybin synergizes with Wnt3a in activation of the Wnt/ $\beta$-catenin signaling pathway through stabilization of intracellular $\beta$-catenin protein. Korean $J$. Microbiol. Biotechnol. 40: 50-56.

33. Meidan VM, Touitou E. 2001. Treatments for androgenetic alopecia and alopecia areata: current options and future prospects. Drugs 61: 53-69.

34. Dinh QQ, Sinclair R. 2007. Female pattern hair loss: current treatment concepts. Clin. Interv. Aging. 2: 189-199.

35. Jain R, Monthakantirat O, Tengamnuay P, De-Eknamkul W. 2016. Identification of a new plant extract for androgenic alopecia treatment using a non-radioactive human hair dermal papilla cell-based assay. BMC Complement. Altern. Med. 16: 18.

36. Harel S, Higgins CA, Cerise JE, Dai Z, Chen JC, Clynes R, et al. 2015. Pharmacologic inhibition of JAK-STAT signaling promotes hair growth. Sci. Adv. 1: e1500973-e1500973.

37. Murkute A, Sahu M, Mali P, Rangari V. 2010. Development and evaluation of formulations of microbial biotransformed extract of tobacco leaves for hair growth potential. Pharmacognosy Res. 2: 300-303.

38. Bureau JP, Ginouves P, Guilbaud J, Roux ME. 2003. Essential oils and low-intensity electromagnetic pulses in the treatment of androgen-dependent alopecia. Adv. Ther. 20: $220-229$. 